\title{
Standard model EFT and the Drell-Yan process at high energy
}

\author{
S. Dawson, ${ }^{1}$ P. P. Giardino, ${ }^{2}$ and A. Ismail ${ }^{3}$ \\ ${ }^{1}$ Department of Physics, Brookhaven National Laboratory, Upton, New York 11973, USA \\ ${ }^{2}$ Instituto de Física Teórica UAM/CSIC, Universidad Autónoma de Madrid, 28049 Madrid, Spain \\ ${ }^{3}$ PITT PACC, University of Pittsburgh, Pittsburgh, Pennsylvania 15260, USA
}

(Received 5 December 2018; published 27 February 2019)

\begin{abstract}
The Drell-Yan process is a copious source of lepton pairs at high energy and is measured with great precision at the Large Hadron Collider (LHC). Barring any new light particles, beyond the Standard Model effects can be studied in Drell-Yan production using an effective field theory. At tree level, new 4-fermion interactions dominate, while at one loop operators modifying 3-gauge boson couplings contribute effects that are enhanced at high energy. We study the sensitivity of the neutral Drell-Yan process to these dimension- 6 operators and compare the sensitivity to that of $W^{+} W^{-}$pair production at the LHC.
\end{abstract}

DOI: $10.1103 /$ PhysRevD.99.035044

\section{INTRODUCTION}

The exploration of the electroweak sector is a major task for the LHC. Without new low scale particles, the only tools available for studying deviations from the SM predictions are effective field theories (EFT). In this approach, low scale physics is assumed to be sensitive to the presence of higher dimension operators. When a complete basis of these operators is constructed, they will affect predictions for many observables, including in Higgs physics $[1,2]$, gauge boson pair production [3-7], top quark production [8-10], and many other processes. The measurements from different processes provide complementary information about the parameters of the EFT and potential insights into the underlying UV physics.

In this work, we consider the effects of a consistent EFT analysis on neutral Drell-Yan production. The DrellYan process is extremely precisely measured at numerous energies, while the Standard Model theoretical predictions exist at next-to-next-to-leading order (NNLO) QCD [1115], along with the resummation of the logarithms $[16,17]$. The QCD corrections have been combined with NLO electroweak effects $[18,19]$ and implemented in the FEWZ code [20-22]. We study neutral Drell-Yan production in the context of the Standard Model effective field theory (SMEFT) [23], where the Higgs boson is assumed to be part of an $S U(2)$ doublet. The effects of the dimension-6 SMEFT operators can potentially be of the

Published by the American Physical Society under the terms of the Creative Commons Attribution 4.0 International license. Further distribution of this work must maintain attribution to the author(s) and the published article's title, journal citation, and DOI. Funded by SCOAP . same magnitude as the higher order Standard Model corrections, and both need to be considered in precision studies. New 4-fermion operators can contribute to $q \bar{q} \rightarrow$ $l^{+} l^{-}$production at tree level and have been extensively studied in the literature [24-28]. Precision measurements at the $Z$-pole place bounds on the strengths of the nonStandard Model 4-fermion operators, and even more stringent constraints come from other low energy measurements including atomic parity violation, deep inelastic scattering and flavor observables.

At the LHC, new information can be gained by looking at high $p_{T}$ (or $m_{l l}$ ) events where the new physics effects are potentially enhanced by contributions of $\mathcal{O}\left(\frac{p_{T}^{2}}{\Lambda^{2}}\right)$. In an EFT approach, the NLO corrections to the EFT contributions are also necessary, and new operators that do not contribute at tree level can have measurable effects. The QCD corrections to Drell-Yan production in the SMEFT are known [29]. The program of electroweak corrections to the SMEFT is in its infancy, however, with results for $H \rightarrow$ $V V$ [30-33], $H \rightarrow b \bar{b}[34,35]$ and $Z \rightarrow f \bar{f}[36,37]$ known. Here we begin the program of one-loop EFT contributions to Drell-Yan production. We consider the one-loop contributions from anomalous 3-gauge boson interactions and compare with the sensitivity to these interactions in $W^{+} W^{-}$ pair production. The sensitivity of Drell-Yan production to oblique corrections at high energy has also been studied in Ref. [38]. We find that while Drell-Yan provides additional information, the impact of anomalous 3-gauge boson interactions is generally more easily observable in $W^{+} W^{-}$ pair production. Additionally, 4-fermion operators which were not considered in Ref. [38] affect the Drell-Yan process at tree level, and unless they are set to zero, as in a universal theory, they can overwhelm the impact of loop corrections from other operators. 
TABLE I. Dimension-6 operators relevant for our study (from [23]). For brevity we suppress fermion chiral indices $L, R . I=1,2,3$ is an $S U(2)$ index. $p, r, s, t=1,2,3$ are generation indices.

\begin{tabular}{|c|c|c|c|c|c|}
\hline $\mathcal{O}_{W}$ & $\epsilon^{I J K} W_{\mu}^{I \nu} W_{\nu}^{J \rho} W_{\rho}^{K \mu}$ & $\mathcal{O}_{\phi D}$ & $\left(\phi^{\dagger} D^{\mu} \phi\right)^{*}\left(\phi^{\dagger} D_{\mu} \phi\right)$ & $\mathcal{O}_{\phi W B}$ & $\left(\phi^{\dagger} \tau^{I} \phi\right)^{*} W_{\mu \nu}^{I} B^{\mu \nu}$ \\
\hline $\mathcal{O}_{p l}^{(3)}$ & $\left(\phi^{\dagger} i \stackrel{\leftrightarrow}{D_{\mu}} \phi\right)\left(\bar{l}_{p}^{\prime} \tau^{I} \gamma^{\mu} l_{r}^{\prime}\right)$ & $\begin{array}{c}\mathcal{O}_{l q}^{(1)} \\
p, r, s, t\end{array}$ & $\left(\bar{l}_{p}^{\prime} \gamma_{\mu} l_{r}^{\prime}\right)\left(\bar{q}_{s}^{\prime} \gamma^{\mu} q_{t}^{\prime}\right)$ & $\begin{array}{c}\mathcal{O}_{l q}^{(3)} \\
p, r, s, t\end{array}$ & $\left(\bar{l}_{p}^{\prime} \gamma_{\mu} \tau^{I} l_{r}^{\prime}\right)\left(\bar{q}_{s}^{\prime} \gamma^{\mu} \tau^{I} q_{t}^{\prime}\right)$ \\
\hline$\underset{p, r, s, t}{\mathcal{O}_{q e}}$ & $\left(\bar{q}_{p}^{\prime} \gamma_{\mu} q_{r}^{\prime}\right)\left(\bar{e}_{s}^{\prime} \gamma^{\mu} e_{t}^{\prime}\right)$ & $\underset{p, r, s, t}{\mathcal{O}_{e u}}$ & $\left(\bar{e}_{p}^{\prime} \gamma_{\mu} e_{r}^{\prime}\right)\left(\bar{u}_{s}^{\prime} \gamma^{\mu} u_{t}^{\prime}\right)$ & $\underset{p, r, s, t}{\mathcal{O}_{e d}}$ & $\left(\bar{e}_{p}^{\prime} \gamma_{\mu} e_{r}^{\prime}\right)\left(\bar{d}_{s}^{\prime} \gamma^{\mu} d_{t}^{\prime}\right)$ \\
\hline$\underset{p, r, s, t}{\mathcal{O}_{l u}}$ & $\left(\bar{l}_{p}^{\prime} \gamma_{\mu} l_{r}^{\prime}\right)\left(\bar{u}_{s}^{\prime} \gamma^{\mu} u_{t}^{\prime}\right)$ & $\mathcal{O}_{p, r}$ & $\left(\bar{l}_{p}^{\prime} \gamma_{\mu} l_{r}^{\prime}\right)\left(\bar{d}_{s}^{\prime} \gamma^{\mu} d_{t}^{\prime}\right)$ & $\underset{p, r, s, t}{\mathcal{O}_{l l}}$ & $\left(\bar{l}_{p}^{\prime} \gamma_{\mu} l_{r}^{\prime}\right)\left(\bar{l}_{s}^{\prime} \gamma^{\mu} l_{t}^{\prime}\right)$ \\
\hline
\end{tabular}

In Sec. II, we review the SMEFT and write the leading order amplitude for Drell-Yan production. Section III shows the results of our NLO calculation involving SMEFT operators. Then, in Sec. IV we demonstrate the impact of SMEFT operators on kinematic distributions in Drell-Yan production and estimate the reach of the LHC in probing these operators. Section V contains our conclusions.

\section{BASICS}

In the SMEFT, new physics is described by a tower of operators,

$$
\mathcal{L}=\mathcal{L}_{\mathrm{SM}}+\Sigma_{k=5}^{\infty} \Sigma_{i=1}^{n} \frac{\mathcal{C}_{i}^{k}}{\Lambda^{k-4}} O_{i}^{k}
$$

The dimension- $k$ operators are constructed from SM fields and the new beyond the SM (BSM) physics effects reside in the coefficient functions, $\mathcal{C}_{i}^{k}$. For large $\Lambda$, it is sufficient to retain only the lowest dimensional operators. The operators have been classified in several different bases, which are related by the equations of motion $[1,23,39,40]$. In this paper we will use the Warsaw basis of Ref. [23] and the convenient implementation of Ref. [41].

Only a few operators contribute to the neutral Drell-Yan process, $f \bar{f} \rightarrow e_{p}^{+} e_{p}^{-}$, at tree level ( $p$ is a generation index). There are new 4-fermion operators, along with operators that shift the tree level relationships among the parameters $[42,43]$. The operators relevant for Drell-Yan production at tree level, along with the operator $\mathcal{O}_{W}$ that is the focus of the next section, are given in Table I. We define $q$ and $l$ to be the $S U(2)_{L}$ quark and lepton doublets, respectively.

The operators in Table I change the form of the kinetic terms in the gauge sector,

$$
\begin{aligned}
\mathcal{L}= & -\frac{1}{4} W^{I, \mu \nu} W_{\mu \nu}^{I}-\frac{1}{4} B^{\mu \nu} B_{\mu \nu} \\
& +\frac{1}{\Lambda^{2}}\left(\mathcal{C}_{\phi W}\left(\phi^{\dagger} \phi\right) W^{I, \mu \nu} W_{\mu \nu}^{I}+\mathcal{C}_{\phi B}\left(\phi^{\dagger} \phi\right) B^{\mu \nu} B_{\mu \nu}\right. \\
& \left.+\mathcal{C}_{\phi W B}\left(\phi^{\dagger} \tau^{I} \phi\right) W^{I, \mu \nu} B_{\mu \nu}\right) .
\end{aligned}
$$

We define "barred" fields, $\bar{W}_{\mu} \equiv\left(1-\mathcal{C}_{\phi W} v^{2} / \Lambda^{2}\right) W_{\mu}$ and $\bar{B}_{\mu} \equiv\left(1-\mathcal{C}_{\phi B} v^{2} / \Lambda^{2}\right) B_{\mu}$ and "barred" gauge couplings, $\bar{g}_{2} \equiv\left(1+\mathcal{C}_{\phi W} v^{2} / \Lambda^{2}\right) g_{2}$ and $\bar{g}_{1} \equiv\left(1+\mathcal{C}_{\phi B} v^{2} / \Lambda^{2}\right) g_{1}$ so that $\bar{W}_{\mu} \bar{g}_{2}=W_{\mu} g_{2}$ and $\bar{B}_{\mu} \bar{g}_{1}=B_{\mu} g_{1}$. The "barred" fields defined in this way have their kinetic terms properly normalized and preserve the form of the covariant derivative. The masses of the $\mathrm{W}$ and $\mathrm{Z}$ fields are then, [41,44],

$$
\begin{aligned}
& M_{W}^{2}=\frac{\bar{g}_{2}^{2} v^{2}}{4}, \\
& M_{Z}^{2}=\frac{\left(\bar{g}_{1}^{2}+\bar{g}_{2}^{2}\right) v^{2}}{4}+\frac{v^{4}}{\Lambda^{2}}\left(\frac{1}{8}\left(\bar{g}_{1}^{2}+\bar{g}_{2}^{2}\right) \mathcal{C}_{\phi D}+\frac{1}{2} \bar{g}_{1} \bar{g}_{2} \mathcal{C}_{\phi W B}\right) .
\end{aligned}
$$

Dimension-6 operators contribute to the decay of the $\mu$ lepton at tree level, changing the relation between the vacuum expectation value, $v$, and the Fermi constant $G_{\mu}$ obtained from the measurement of the $\mu$ lifetime,

$$
\begin{aligned}
G_{\mu} & =\frac{1}{\sqrt{2} v^{2}}-\frac{1}{2 \sqrt{2} \Lambda^{2}}\left(\underset{1,2,2,1}{\mathcal{C}_{l l}}+\underset{2,1,1,2}{\mathcal{C}_{l l}}\right)+\frac{\sqrt{2}}{2 \Lambda^{2}}\left(\mathcal{C}_{\phi l}{ }_{1,1}^{(3)}+\underset{2,2}{\mathcal{C}_{\phi l}{ }^{(3)}}\right) \\
& \equiv \frac{1}{\sqrt{2} v^{2}}-\frac{1}{\sqrt{2} \Lambda^{2}} \mathcal{C}_{l l}+\frac{\sqrt{2}}{\Lambda^{2}} \mathcal{C}_{\phi l}^{(3)}
\end{aligned}
$$

where we assume flavor universality of the coefficients in the last line above.

We choose the $G_{\mu}$ scheme, where we take the physical input parameters to be

$$
\begin{aligned}
G_{\mu} & =1.1663787(6) \times 10^{-5} \mathrm{GeV}^{-2} \\
M_{Z} & =91.1876 \pm .0021 \mathrm{GeV} \\
M_{W} & =80.385 \pm .015 \mathrm{GeV}
\end{aligned}
$$

Using an input basis of $M_{W}, M_{Z}$ and $G_{\mu}$ [43], the effective fermion $-Z / \gamma$ interactions are 


$$
\begin{aligned}
L= & \frac{2 M_{W}}{v} \sqrt{1-\frac{M_{W}^{2}}{M_{Z}^{2}}} Q_{f}\left\{1-\frac{c_{W}}{s_{W}} v^{2} \mathcal{C}_{\phi W B}-\frac{1}{4} \frac{c_{W}^{2}}{s_{W}^{2}} v^{2} \mathcal{C}_{\phi D}\right\} \bar{f} \gamma^{\mu} f A_{\mu}+\frac{2 M_{Z}}{v}\left\{T_{3}^{f}-Q_{f}\left(1-\frac{M_{W}^{2}}{M_{Z}^{2}}\right)\left(1-\frac{c_{W}}{s_{W}} v^{2} \mathcal{C}_{\phi W B}\right)\right. \\
& \left.-\frac{v^{2}}{4}\left(T_{3}^{f}-Q_{f}\left(1+\frac{M_{W}^{2}}{M_{Z}^{2}}\right)\right) \mathcal{C}_{\phi D}-\frac{v^{2}}{2}\left(\mathcal{C}_{\phi f}^{(1)}-2 T_{3}^{f} \mathcal{C}_{\phi f}^{(3)}\right)\right\} \bar{f} \gamma^{\mu} P_{L} f Z_{\mu} \\
& +\frac{2 M_{Z}}{v}\left\{-Q_{f}\left(1-\frac{M_{W}^{2}}{M_{Z}^{2}}\right)\left(1-\frac{c_{W}}{s_{W}} v^{2} \mathcal{C}_{\phi W B}\right)+\frac{v^{2}}{4} Q_{f}\left(1+\frac{M_{W}^{2}}{M_{Z}^{2}}\right) \mathcal{C}_{\phi D}-\frac{v^{2}}{2} \mathcal{C}_{\phi f}\right\} \bar{f} \gamma^{\mu} P_{R} f Z_{\mu},
\end{aligned}
$$

where $c_{W}=M_{W} / M_{Z}, s_{W}=\sqrt{1-c_{W}^{2}}, T_{3}^{f}= \pm \frac{1}{2}, P_{L, R}=$ $\frac{\left(1 \pm \gamma_{5}\right)}{2}$, and $f=q, l$ for left-handed fermions and $f=u, d, e$ for right-handed fermions. (We omit the generation indices for simplicity.)

The tree level SM result for $q_{i} \bar{q}_{i} \rightarrow e_{p}^{+} e_{p}^{-}$receives corrections from s-channel $Z / \gamma$ exchange,

$$
A_{X Y}^{\mathrm{SM}}=M_{X Y}\left\{\frac{4 M_{W}^{2}}{v^{2}} s_{W}^{2} \frac{Q_{l} Q_{q}}{s}+\frac{g_{q X} g_{l Y}}{s-M_{Z}^{2}}\right\},
$$

where $X, Y=L, R$,

$$
\begin{aligned}
& g_{f L}=\frac{2 M_{Z}}{v}\left[T_{3}^{f}-Q_{f}\left(1-\frac{M_{W}^{2}}{M_{Z}^{2}}\right)\right] \\
& g_{f R}=\frac{2 M_{Z}}{v}\left[-Q_{f}\left(1-\frac{M_{W}^{2}}{M_{Z}^{2}}\right)\right],
\end{aligned}
$$

and

$$
M_{X Y} \equiv\left(\bar{f}_{r} \gamma_{\mu} P_{X} f_{r}\right)\left(\bar{e}_{p} \gamma^{\mu} P_{Y} e_{p}\right) .
$$

The tree level SMEFT amplitudes instead read

$$
\begin{aligned}
A_{X Y}^{\mathrm{SMEFT}}= & A_{X Y}^{\mathrm{SM}}-\frac{v^{2}}{\Lambda^{2}} M_{X Y}\left\{\frac{8 M_{W}^{2}}{v^{2}}\left(s_{W} c_{W} C_{\phi W B}+\frac{c_{W}^{2}}{4} C_{\phi D}\right)\right. \\
& \times \frac{Q_{l} Q_{q}}{s}+\frac{1}{2} C_{\phi D} \frac{g_{q X} g_{l Y}}{s-M_{Z}^{2}}-\frac{2 M_{Z}}{v}\left(s_{W} c_{W} C_{\phi W B}\right. \\
& \left.\left.+\frac{c_{W}^{2}}{2} C_{\phi D}\right) \frac{g_{q X} Q_{l}+g_{l Y} Q_{q}}{s-M_{Z}^{2}}\right\}+A_{X Y, q}^{4-\text { fermions }}, \quad(10)
\end{aligned}
$$

where

$$
\begin{aligned}
& A_{L L, q}^{4 \text {-fermions }}=\frac{M_{X Y}}{\Lambda^{2}}\left(\underset{2,2,1,1}{C_{l q}^{(1)}-2 T_{3}^{q} \mathcal{C}_{l q}^{(3)}}\right) \\
& A_{L R, q}^{4 \text {-fermions }}=\frac{M_{X Y}}{\Lambda^{2}}\left(\underset{1,1,2,2}{\mathcal{C}_{q e}}\right) \\
& A_{R L, u(d)}^{4 \text {-fermions }}=\frac{M_{X Y}}{\Lambda^{2}}\left(\mathcal{C}_{l u(d)}\right) \\
& A_{R R, u(d)}^{4 \text {-fermions }}=\frac{M_{X Y}}{\Lambda^{2}}\left(\mathcal{C}_{e u(d)}\right) .
\end{aligned}
$$

The 4-fermion operators give contributions that grow with energy relative to the SM contributions. and the phenomenological effects have been examined in Refs. [25,26]. Our results are in agreement with these references.

\section{NLO AMPLITUDES}

At one loop, there are contributions to Drell-Yan from new operators not contributing at tree level. We focus on $O_{W}$. This operator is particularly interesting because it is strongly restricted from $f \bar{f} \rightarrow W^{+} W^{-}$both at LEP and at the LHC as its effects grow with energy. The sensitivity to $\mathcal{C}_{W}$ from global fits to LHC measurements and to LEP data has been found in Refs. [7,45-47], and is roughly,

$$
-0.17<\mathcal{C}_{W}\left(\frac{1 \mathrm{TeV}}{\Lambda^{2}}\right)^{2}<0.18
$$

It has been speculated [10] that because of the large cross section and precision of the measurements that high energy Drell-Yan could also yield a precise determination of $\mathcal{C}_{W}$. The fits of Refs. [7,45-47] include the measurement of the Drell-Yan process on the Z-peak.

The diagrams of Fig. 1 give contributions to the left-hand amplitudes. The complete one-loop amplitudes proportional to $\mathcal{C}_{W}$ are available in the online archive associated with our results, and the energy enhanced (relative to the SM) contributions are

$$
\begin{aligned}
& A_{L L, u}^{\mathrm{NLO}}=A_{L L, u}^{\mathrm{SMEFT}}\left(1-\left[\frac{3 s v^{2}}{\Lambda^{2} M_{Z}^{2}\left(1+2 c_{W}^{2}\right)}\right]\left\{\frac{g^{3} \mathcal{C}_{W}}{32 \pi^{2}}\right\}\right) \\
& A_{L L, d}^{\mathrm{NLO}}=A_{L L, d}^{\mathrm{SMEFT}}\left(1+\left[\frac{3 s v^{2}}{\Lambda^{2} M_{Z}^{2}\left(1-4 c_{W}^{2}\right)}\right]\left\{\frac{g^{3} \mathcal{C}_{W}}{32 \pi^{2}}\right\}\right) .
\end{aligned}
$$

These amplitudes include the NLO shifts of the input parameters $[37,48]$ as well as the one-loop diagrams proportional to $\mathcal{C}_{W}$, consisting of both vertex and propagator corrections. When the contributions from the input parameter shifts and the one-loop diagrams are added together, the divergences cancel completely in the NLO amplitudes as expected. 

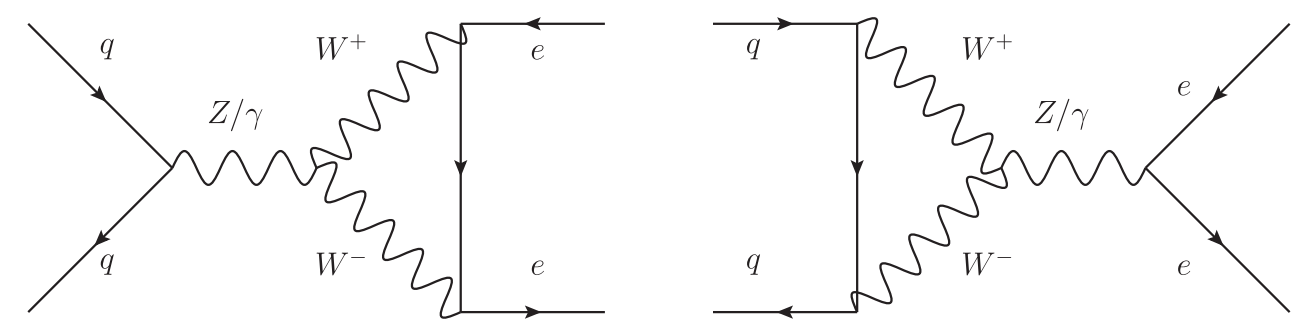

FIG. 1. Vertex corrections proportional to $\mathcal{C}_{W}$.

\section{RESULTS}

We now use the amplitudes of Secs. II and III to calculate the effects of SMEFT operators on kinematic distributions in Drell-Yan production. Current and future precision measurements of $p p \rightarrow e_{p}^{+} e_{p}^{-}$will constrain not only 4-fermion operators involving quarks and leptons, but also purely bosonic operators that contribute at loop level. We concentrate on the effect of the latter, that would dominate in a universal theory [49] or one in which the sizes of the coefficients of BSM 4-fermion operators involving first and second generation quarks and leptons are small.

In Fig. 2, we show the fractional modification of the dilepton mass distribution due to different bosonic operators. We include not only the operators $\mathcal{O}_{\phi W B}$ and $\mathcal{O}_{W}$, which cause loop effects and are the focus of our calculation, but also one of the 4-fermion operators that affects Drell-Yan production at tree level, for comparison. $\mathcal{C}_{\phi W B}$ corresponds to the $S$ parameter $S[50,51]$ and is constrained to be

$$
-0.004<\mathcal{C}_{\phi W B}\left(\frac{1 \mathrm{TeV}}{\Lambda}\right)^{2}<0.006
$$

where we take our limit from the one-parameter fit of the Gfitter Collaboration [52]. The bound from the LEP

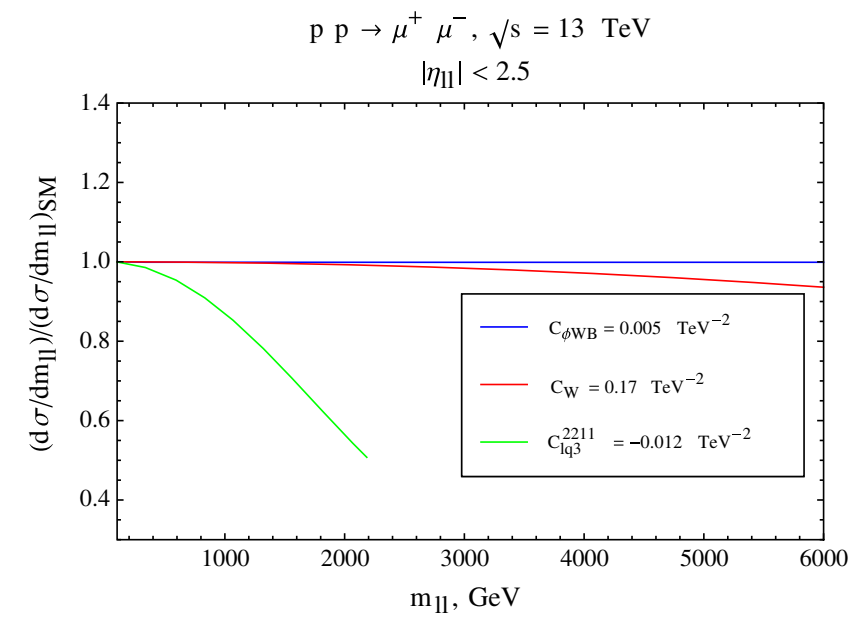

precision electroweak data dominates the global fits of Refs. [7,45-47]. $C_{W}$ is bounded by $W^{+} W^{-}$production, as described in Sec. III. The 4-fermion operators are limited by existing Drell-Yan measurements [53], and of the many potential operators which contribute, the best constrained operator is $\mathcal{O}_{l q}^{(3)}$, whose coefficient is limited by a oneparameter fit to be

$$
\begin{aligned}
-0.012< & \mathcal{C}_{l q}^{(3)}\left(\frac{1 \mathrm{TeV}}{\Lambda}\right)^{2}<0.0047,1,1 \\
& \times(\text { Single parameter fit }[53])
\end{aligned}
$$

The results of the global SMEFT fit of Ref. [47] find considerably less stringent bounds on $C_{l q}^{(3)}$ due to large correlations between the effects of different operators.

The left panel shows the effects of these operators at the LHC, while the right panel shows them at a future $100 \mathrm{TeV}$ collider. For the 4-fermion operator, the EFT loses validity at high invariant mass because the neglected dimension- 8 operators become important, and it is no longer appropriate to treat the EFT contribution as a small correction to the SM amplitude. We have cut off the associated curves where $A_{L L, q}^{4 \text {-fermions }}=A_{L L}^{\mathrm{SM}} / 2$, which occurs at the center of mass

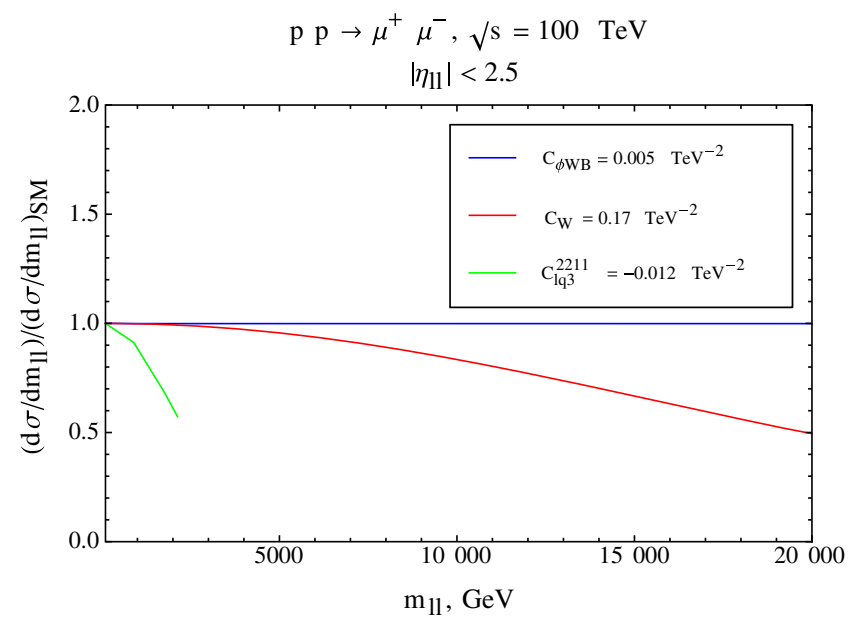

FIG. 2. The ratio of the differential cross section as a function of dilepton mass in the SMEFT to that in the SM. In each curve, one operator coefficient is turned on at a time, while the others are set to zero. The sizes of the operators are taken to be at their current bounds. The left (right) plot shows the distribution for 14 (100) TeV. The results for the 4-fermion operator are cut off where the BSM contribution becomes similar in magnitude to the SM amplitude, as detailed in the text. 
$\mathrm{p} \mathrm{p} \rightarrow \mu^{+} \mu^{-}, \sqrt{ } \mathrm{s}=13 \mathrm{TeV}$

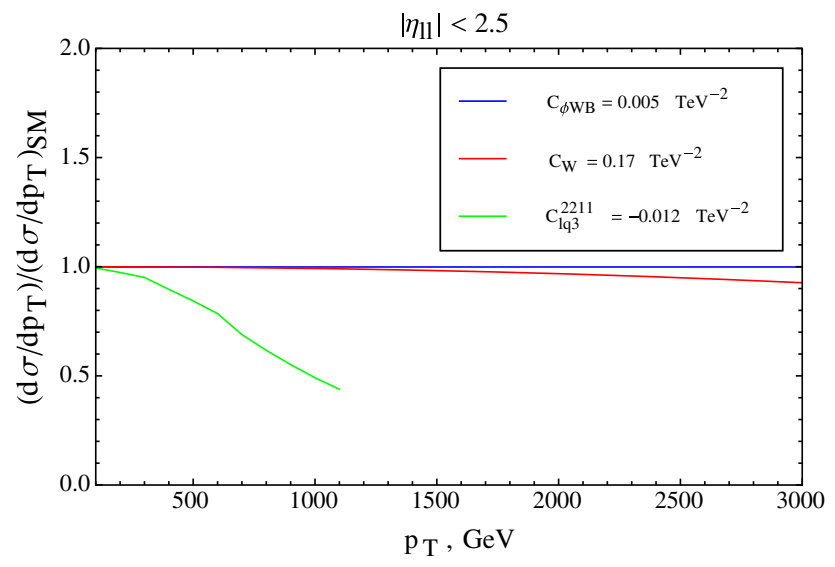

$\mathrm{p} \mathrm{p} \rightarrow \mu^{+} \mu^{-}, \sqrt{ } \mathrm{s}=100 \mathrm{TeV}$

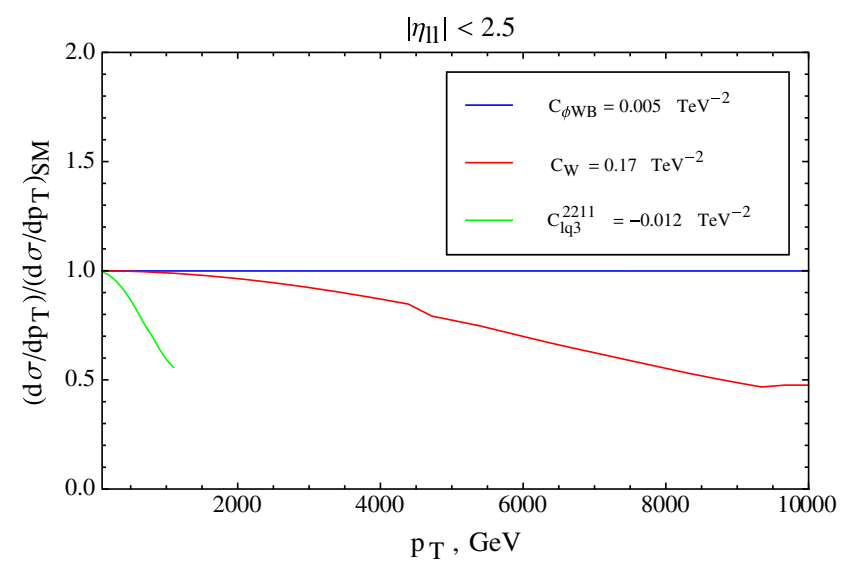

FIG. 3. Same as Fig. 2, but for the $p_{T}$ distribution.

energy $m_{\ell \ell}^{*}=2.2 \mathrm{TeV}$. Nevertheless, it is clear that in a nonuniversal theory, new 4-fermion operators can change Drell-Yan production much more than purely bosonic operators, even those such as $\mathcal{C}_{\phi W B}$ which contribute at tree level. At $100 \mathrm{TeV}$, where Drell-Yan could be potentially measured up to $m_{\ell \ell}=20 \mathrm{TeV}$ [54], the operator $\mathcal{C}_{W}$ at its current $2 \sigma$ limit could provide up to a $50 \%$ deviation in the number of events at high energy. The effects are similarly sized for the $p_{T}$ distribution, as shown in Fig. 3.

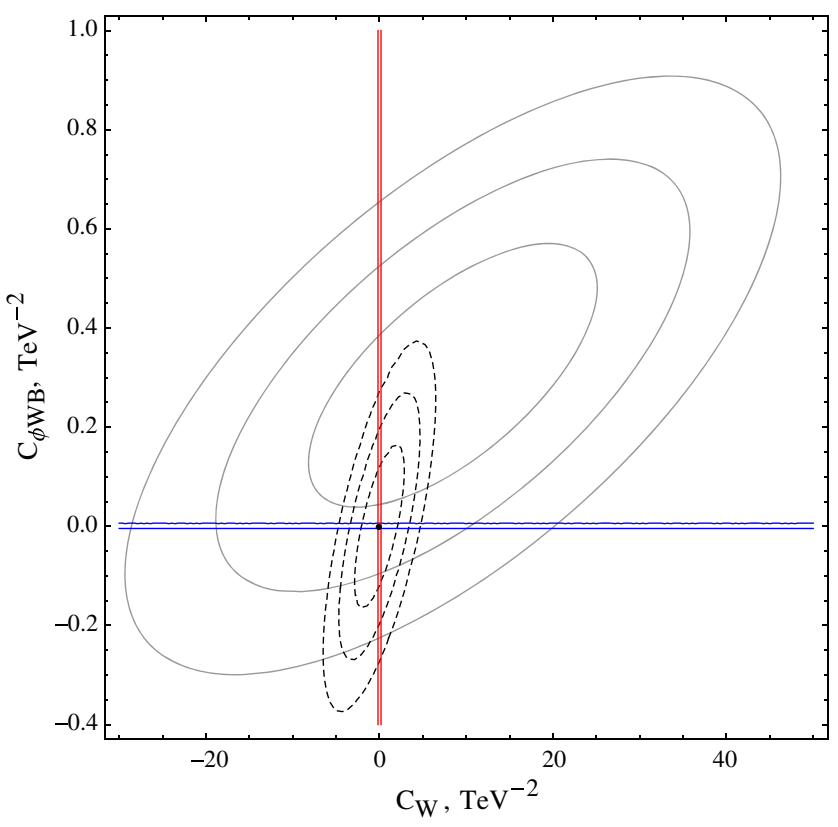

FIG. 4. Contours in the $\mathcal{C}_{W}-\mathcal{C}_{\phi W B}$ plane resulting from a fit to the CMS measurement of Drell-Yan. The contours indicate $68.3 \%, 95.4 \%$, and $99.7 \%$ confidence contours around the best-fit point. Solid contours are for the current $8 \mathrm{TeV}$ measurement [55], while dashed contours are for the HL-LHC. The Standard Model is indicated at $(0,0)$. The region between the blue (red) lines is allowed by the current limits of Eq. (14) (Eq. (12).
Again, the 4-fermion operator contributions become large at high $p_{T}$, and we avoid any phase space corresponding to an invariant mass higher than $m_{\ell \ell}^{*}$ by cutting off the corresponding curves at $p_{T}=m_{\ell \ell}^{*} / 2=1.1 \mathrm{TeV}$.

From our derived effects of new operators on the DrellYan $m_{\ell \ell}$ distribution, we can place limits on the sizes of these operators from existing measurements of Drell-Yan production. We use the $8 \mathrm{TeV}$ CMS measurement [55], which goes up to $2 \mathrm{TeV}$ in the dilepton invariant mass. Using the CMS data above $240 \mathrm{GeV}$ and neglecting correlated uncertainties among different bins, ${ }^{1}$ we construct a $\chi^{2}$ function expressing the goodness of fit between the observed data and the prediction for arbitrary sizes of the SMEFT operators $\mathcal{O}_{W}$ and $\mathcal{O}_{\phi W B}$. We consider only how the new operators affect the ratio of the data to theory and are not sensitive to the overall normalization of the DrellYan invariant mass distribution. In Fig. 4, we show the allowed region in the plane of the sizes of these two operators. The $13 \mathrm{TeV}$ measurement [56] using $2.8 \mathrm{fb}^{-1}$ of data already has comparable uncertainties in bins going out to $3 \mathrm{TeV}$ in $m_{\ell \ell}$, and we also show a projection for the high luminosity upgrade of the LHC assuming that statistical uncertainties scale as $1 / \sqrt{L}$ such that the uncertainties in each bin would be limited only by systematics, which are currently around 5\%. The currently allowed region in the plane is significantly larger than that allowed by constraints from electroweak precision and $W W$ production on the operator coefficients $\mathcal{C}_{\phi W B}$ and $\mathcal{C}_{W}$, respectively. However, as both $\mathcal{O}_{\phi W B}$ and $\mathcal{O}_{W}$ contribute to Drell-Yan, an external constraint on one of the operator coefficients in conjunction with a measurement of the Drell-Yan differential distribution can constrain the other better.

\footnotetext{
${ }^{1}$ At high invariant mass, in the region where the SMEFT operators are expected to have the greatest effect, the uncertainty is dominantly statistical.
} 


\section{CONCLUSION}

In the absence of new light physics, the EFT approach provides a parametrization of BSM effects in terms of higher dimension operators. Given the minute precision with which many processes can be measured at the LHC, especially at high luminosity, it is of use to know the loop contributions of EFT operators to SM physics. In this work, we have evaluated some of the these corrections in the SMEFT for Drell-Yan production.

Notably, operators which do not contribute at tree level to Drell-Yan can have sizable contributions at one loop. We have shown that at the upper end of the energy range that can be probed at the LHC, the effect of the operator $\mathcal{O}_{W}$ can be several percent, or even up to $50 \%$ at a future $100 \mathrm{TeV}$ collider. Over the lifetime of the LHC, statistical uncertainties will go down significantly as more data are collected, significantly increasing the sensitivity of precision DrellYan measurements in the region where new physics operators have the greatest effect. As a consequence, future measurements of Drell-Yan offer the possibility to constrain operators such as $\mathcal{O}_{W}$ even though their contributions are only at one loop. While gauge boson production is still a more sensitive probe of $\mathcal{O}_{W}$, the effects which we have computed here must be taken into account to ensure consistency in a full NLO fit to the coefficients of SMEFT operators.

\section{ACKNOWLEDGMENTS}

S. D. is supported by the U.S. Department of Energy under Grant Contract No. DE-SC0012704. P. P. G. is supported by the Spanish Research Agency (Agencia Estatal de Investigacion) through the Contract No. FPA201678022-P and IFT Centro de Excelencia Severo Ochoa under Grant No. SEV-2016-0597. A. I. is supported by the U.S. Department of Energy under Grant Contract No. DESC0015634 and by Pittsburgh Particle Physics, Astrophysics and Cosmology Center (PITT PACC). Digital data related to our results can be found at https://quark.phy.bnl.gov/Digital_Data_Archive/dawson/ drellyan_18.
[1] G. F. Giudice, C. Grojean, A. Pomarol, and R. Rattazzi, J. High Energy Phys. 06 (2007) 045.

[2] I. Brivio and M. Trott, Phys. Rept. 793, 1 (2019).

[3] J. Baglio, S. Dawson, and I. M. Lewis, Phys. Rev. D 96, 073003 (2017).

[4] A. Azatov, R. Contino, C. S. Machado, and F. Riva, Phys. Rev. D 95, 065014 (2017).

[5] Z. Zhang, Phys. Rev. Lett. 118, 011803 (2017).

[6] C. Grojean, M. Montull, and M. Riembau, arXiv:1810.05149.

[7] A. Alves, N. Rosa-Agostinho, O. J. P. Eboli, and M. C. Gonzalez-Garcia, Phys. Rev. D 98, 013006 (2018).

[8] D. Barducci et al., arXiv:1802.07237.

[9] C. Degrande, J. M. Gerard, C. Grojean, F. Maltoni, and G. Servant, J. High Energy Phys. 07 (2012) 036; 03 (2013) 032(E).

[10] M. Farina, C. Mondino, D. Pappadopulo, and J. T. Ruderman, J. High Energy Phys. 01 (2019) 231.

[11] C. Anastasiou, L. J. Dixon, K. Melnikov, and F. Petriello, Phys. Rev. Lett. 91, 182002 (2003).

[12] A. Gehrmann-De Ridder, T. Gehrmann, E. W. N. Glover, A. Huss, and T. A. Morgan, J. High Energy Phys. 11 (2016) 094; 10 (2018) 126(E).

[13] A. Gehrmann-De Ridder, T. Gehrmann, E. W. N. Glover, A. Huss, and T. A. Morgan, J. High Energy Phys. 07 (2016) 133.

[14] A. Karlberg, E. Re, and G. Zanderighi, J. High Energy Phys. 09 (2014) 134.

[15] S. Hoche, Y. Li, and S. Prestel, Phys. Rev. D 91, 074015 (2015).

[16] W. Bizo, X. Chen, A. Gehrmann-De Ridder, T. Gehrmann, N. Glover, A. Huss, P. F. Monni, E. Re, L. Rottoli, and P. Torrielli, J. High Energy Phys. 12 (2018) 132.
[17] S. Alioli, C. W. Bauer, C. Berggren, F. J. Tackmann, and J. R. Walsh, Phys. Rev. D 92, 094020 (2015).

[18] S. Dittmaier and M. Huber, J. High Energy Phys. 01 (2010) 060 .

[19] U. Baur, O. Brein, W. Hollik, C. Schappacher, and D. Wackeroth, Phys. Rev. D 65, 033007 (2002).

[20] Y. Li and F. Petriello, Phys. Rev. D 86, 094034 (2012).

[21] R. Gavin, Y. Li, F. Petriello, and S. Quackenbush, Comput. Phys. Commun. 182, 2388 (2011).

[22] C. M. Carloni Calame, G. Montagna, O. Nicrosini, and A. Vicini, J. High Energy Phys. 10 (2007) 109.

[23] B. Grzadkowski, M. Iskrzynski, M. Misiak, and J. Rosiek, J. High Energy Phys. 10 (2010) 085.

[24] A. Falkowski, M. González-Alonso, and K. Mimouni, J. High Energy Phys. 08 (2017) 123.

[25] L. Berthier and M. Trott, J. High Energy Phys. 05 (2015) 024.

[26] J. de Blas, M. Chala, and J. Santiago, Phys. Rev. D 88, 095011 (2013).

[27] V. Cirigliano, M. Gonzalez-Alonso, and M. L. Graesser, J. High Energy Phys. 02 (2013) 046.

[28] M. Carpentier and S. Davidson, Eur. Phys. J. C 70, 1071 (2010).

[29] S. Alioli, W. Dekens, M. Girard, and E. Mereghetti, J. High Energy Phys. 08 (2018) 205.

[30] S. Dawson and P. P. Giardino, Phys. Rev. D 97, 093003 (2018).

[31] S. Dawson and P. P. Giardino, Phys. Rev. D 98, 095005 (2018).

[32] C. Hartmann and M. Trott, Phys. Rev. Lett. 115, 191801 (2015). 
[33] A. Dedes, M. Paraskevas, J. Rosiek, K. Suxho, and L. Trifyllis, J. High Energy Phys. 08 (2018) 103.

[34] R. Gauld, B. D. Pecjak, and D. J. Scott, Phys. Rev. D 94, 074045 (2016).

[35] R. Gauld, B. D. Pecjak, and D. J. Scott, J. High Energy Phys. 05 (2016) 080.

[36] C. Hartmann, W. Shepherd, and M. Trott, J. High Energy Phys. 03 (2017) 060.

[37] S. Dawson and A. Ismail, Phys. Rev. D 98, 093003 (2018).

[38] M. Farina, G. Panico, D. Pappadopulo, J. T. Ruderman, R. Torre, and A. Wulzer, Phys. Lett. B 772, 210 (2017).

[39] W. Buchmuller and D. Wyler, Nucl. Phys. B268, 621 (1986).

[40] K. Hagiwara, S. Ishihara, R. Szalapski, and D. Zeppenfeld, Phys. Rev. D 48, 2182 (1993).

[41] A. Dedes, W. Materkowska, M. Paraskevas, J. Rosiek, and K. Suxho, J. High Energy Phys. 06 (2017) 143.

[42] A. Falkowski, Pramana 87, 39 (2016).

[43] I. Brivio and M. Trott, J. High Energy Phys. 07 (2017) 148; 05 (2018) 136(A).

[44] R. Alonso, E. E. Jenkins, A. V. Manohar, and M. Trott, J. High Energy Phys. 04 (2014) 159.
[45] A. Biekotter, T. Corbett, and T. Plehn, arXiv:1812.07587.

[46] E. da Silva Almeida, A. Alves, N. Rosa Agostinho, O. J. P. Eboli, and M. C. Gonzalez-Garcia, Phys. Rev. D 99, 033001 (2019).

[47] M. Bjorn and M. Trott, Phys. Lett. B 762, 426 (2016).

[48] C.-Y. Chen, S. Dawson, and C. Zhang, Phys. Rev. D 89, 015016 (2014).

[49] J. D. Wells and Z. Zhang, J. High Energy Phys. 06 (2016) 122.

[50] M. E. Peskin and T. Takeuchi, Phys. Rev. Lett. 65, 964 (1990).

[51] M. E. Peskin and T. Takeuchi, Phys. Rev. D 46, 381 (1992).

[52] J. Haller, A. Hoecker, R. Kogler, K. Mönig, T. Peiffer, and J. Stelzer, Eur. Phys. J. C 78, 675 (2018).

[53] A. Greljo and D. Marzocca, Eur. Phys. J. C 77, 548 (2017).

[54] M. L. Mangano et al., CERN Yellow Report No. CERNTH-2016-112, pp. 1-254, 2017.

[55] V. Khachatryan et al. (CMS Collaboration), Eur. Phys. J. C 75, 147 (2015).

[56] Technical Report No. CMS-PAS-SMP-16-019, CERN, Geneva, 2017. 